\title{
EDITORIAL
}

\section{Optical fiber-based devices and applications}

\author{
Perry Ping SHUM $(\bowtie)^{1}$, Jonathan C. KNIGHT ${ }^{2}$, Jesper LAGGGAARD ${ }^{3}$, Dora Juan Juan HU ${ }^{4}$ \\ 1 School of Electrical and Electronic Engineering, Nanyang Technological University, Singapore 639798, Singapore \\ 2 Centre for Photonics and Photonic Materials and Department of Physics, University of Bath, Bath BA2 7AY, UK \\ 3 Department of Photonics Engineering, Technical University of Denmark, Kongens Lyngby 2800, Denmark \\ 4 RF and Optical Department, Institute for Infocomm Research, Agency for Science, Technology and Research (A*STAR), \\ Singapore 138668, Singapore
}

(C) Higher Education Press and Springer-Verlag 2009

Optical fiber technology has undergone tremendous growth and development over the last 40 years. Optical fibers constitute an information super highway and are vital in enabling the proliferating use of the Internet. Optical fiber is also an enabling technology which can find applications in sensing, imaging, biomedical, machining, etc. There have been a few milestones in the advancement of optical fiber technology. Firstly, the invention and development of the laser some 50 years ago made optical communications possible. Secondly, the fabrication of low-loss optical fibers has been a key element to the success of optical communication. Thirdly, continuous advances and development in the design and implementation of fiber-based devices such as fiber gratings and fiber amplifiers continue to add competence and open up new applications for optical fiber technology. Optical fibers can act simultaneously as sensing elements and signal transmission media, so that both distributed sensing and remote sensing can be realized. Optical fiber-based sensors possess many advantages such as light weight, immunity to radio frequency interference and electromagnetic interference, high sensitivity, compact size, corrosion resistance and low cost.

Besides conventional optical fibers, microstructured optical fibers (MOFs) have received enormous attention since their first demonstration a decade ago. These specialty fibers have air holes, usually arranged in a periodic configuration, running down their length; the air holes provide unprecedented design freedom in shaping the optical properties. Recent results have shown that MOFs can be designed with tailorable properties including dispersion, nonlinearity, birefringence, cutoff condition, etc; they can be used to generate kilowatt-level fiber lasers and to deliver high powers; to make a supercontinuum source, etc. The sensing applications are investigated and developed for gases, liquids, biochemical detection, etc. Besides silica, other alternative materials such as polymers and soft glasses are investigated. The optical fibers can be tapered to sub-micron scale: nanofibers are emerging as an enabling technology for developing micro- or nano-photonic components/devices ranging from interferometers, filters, lasers to sensors.

This special issue, Frontiers of Optical Fiber Devices and Applications, contains contributions from prominent professors and researchers showcasing the latest results in the field of development and application of optical fiber technology. The aim of the special issue is to provide a broad snapshot of the current state-of-the-art as well as the prospects in the field.

Received November 20, 2009

E-mail: epshum@ntu.edu.cn 